\title{
Visions et spectres dans la littérature norroise : aperçus sur la culture germanique ancienne
}

Erscheinungen und Geister in der altnordischen Literatur und in der frühgermanischen Kultur

Visions and ghosts in Old Norse literature and Ancient Germanic culture

Daniel Lacroix

\section{(2) OpenEdition}

Journals

Édition électronique

URL : https://journals.openedition.org/ceg/11381

DOI : $10.4000 /$ ceg. 11381

ISSN : 2605-8359

Éditeur

Presses Universitaires de Provence

Édition imprimée

Date de publication : 1 avril 2012

Pagination : 13-23

ISBN : 0751-4239

ISSN : 0751-4239

Référence électronique

Daniel Lacroix, «Visions et spectres dans la littérature norroise : aperçus sur la culture germanique ancienne », Cahiers d'Études Germaniques [En ligne], 62 | 2012, mis en ligne le 12 octobre 2020, consulté le 04 juin 2021. URL : http://journals.openedition.org/ceg/11381 ; DOI : https://doi.org/ 10.4000/ceg. 11381 


\title{
Visions et spectres dans la littérature norroise : aperçus sur la culture germanique ancienne
}

\author{
Daniel LACROIX \\ Université de Toulouse 2 - Le Mirail
}

La culture germanique ancienne, c'est-à-dire païenne, perdure en Scandinavie jusqu'au $\mathrm{XI}^{\mathrm{e}}$ siècle. Dans celle-ci, la conception ordinaire du monde fait apparaître un double plan de réalité : d'une part, le monde habité par les vivants, d'autre part, un autre monde la plupart du temps invisible où se côtoient dieux et divinités, morts et esprits, créatures et forces placés dans un arrière-plan, un envers du décor quotidien, restant proche et familier malgré son étrangeté. Ces conceptions ont certainement couru dans toute la Germanie avant la christianisation, et ont perduré ensuite sous forme de superstitions ou de croyances populaires inscrites dans la mémoire profonde des peuples germaniques jusqu'à une époque récente, au cœur de nos folklores. Nous nous proposons donc d'éclairer ces conceptions anciennes à partir de sources textuelles. Comme on le sait, les croyances germaniques anciennes ont été conservées de façon exceptionnellement précise en Europe du nord, sous la forme d'œuvres littéraires s'étalant de l'an mil au XIV ${ }^{\mathrm{e}}$ siècle. Pour les plus anciennes, toutes ou presque ont été recueillies à partir du $\mathrm{XII}^{\mathrm{e}}$ siècle par les clercs islandais qui ont consigné sur parchemin les productions de leurs ancêtres, accomplissant parfois un véritable travail d'édition ou de critique ${ }^{1}$. C'est en Islande également qu'après le XIV siècle, à la fin de cette période de grande activité intellectuelle, les manuscrits ont été tant bien que mal conservés dans cette île lointaine qui a joué un rôle de conservatoire des antiquités germaniques. Notre corpus est vaste puisqu'il part des poèmes eddiques, englobe au passage l'Edda de Snorri Sturluson et s'étend à une bonne partie de la littérature de sagas - donc l'ensemble de la littérature norroise du Moyen Âge.

La lecture de ces textes nous fournit, en effet, quantité d'éléments nous permettant de comprendre comment s'organisent ces deux plans de la réalité

1 Voir à ce sujet, R. BOYER, La vie religieuse en Islande (1116-1264), d'après la Sturlunga saga et les Sagas des Évêques, Paris, Fondation Singer-Polignac, 1979; L'Islande médiévale, Paris, Les Belles Lettres, 2001, p. 159-216. 
dans les conceptions des anciens Germains. Il est même possible de s'en faire une idée très précise, le sujet étant en vérité extrêmement riche et excédant de beaucoup le cadre de ce travail. Nous nous contenterons donc de dégager quelques grandes lignes à partir d'exemples précis qui nous semblent significatifs ${ }^{2}$.

\section{Contexte général}

Le monde, dans les conceptions païennes anciennes, est conçu de telle manière qu'au-delà de l'univers naturel, des forces et des entités de toutes natures gravitent autour des hommes, entrent fréquemment en rapport avec eux et interfèrent continuellement dans le cours de leur existence. C'est donc un monde où l'homme n'est jamais seul face à la nature et à lui-même, et où il n'est jamais libre de surcroît. Même si les dieux n'ont pas ici l'importance qu'ils ont pu avoir dans d'autres parties de l'ère indo-européenne, d'autres êtres peuplent le voisinage invisible de l'homme. Le monde visible et son envers constituent un même espace de proximité, où la circulation est possible selon des modalités diverses, sans qu'on puisse appliquer là nos catégories modernes, comme celle d'au-delà immatériel ou de monde spirituel. Les catégories même de merveilleux ou de fantastique sont en l'occurrence douteuses, tout au moins à une époque ancienne, quand l'ensemble des deux plans de la réalité était perçu de façon terre-à-terre, réaliste, naturelle.

Malgré tout, il ne faut pas oublier que nos sources sont littéraires et que les clercs islandais ont travaillé plusieurs siècles après les scaldes païens, ou après le temps de la colonisation de l'Islande. Chez eux, par contre, et notamment dans certaines sagas, la place accordée à l'envers du décor, ce monde du double, est telle, à partir de motifs parfois ressassés jusqu'à l'usure, qu'il est possible que nos catégories d'analyse littéraire redeviennent finalement pertinentes. C'est un point sur lequel, d'ailleurs, nous ne suivons pas entièrement nos devanciers, Régis Boyer ou Claude Lecouteux ${ }^{3}$. Il nous semble que la littérature norroise ne doit pas s'interpréter de façon réductrice, comme si elle n'avait eu pour fonction que de recueillir des croyances et de les mettre en forme dans des documents de premier plan pour les futurs historiens des religions, des anthropologues ou des spécialistes des croyances populaires dans la longue durée. En effet, la mise en forme littéraire est ici comme partout ailleurs un acte d'auteur qui fait sens dans un genre littéraire

2 Pour un tour d'horizon plus complet, nous renvoyons à l'étude fondamentale de R. BOYER, Le monde du double : La magie chez les anciens Scandinaves, Paris, Berg International, 1986.

3 Dans la riche production de cet auteur, nous renvoyons tout spécialement aux ouvrages suivants : Fantômes et revenants au Moyen Age, Paris, Imago, (1ère éd.1996) 2009; Les Esprits et les Morts : Croyances médiévales, Paris, 1990 (en collaboration avec Philippe MARCQ); Mondes parallèles : L'Univers des croyances du Moyen Âge, Paris, Champion, 2007. 
donné - par exemple la strophe scaldique ou la saga islandaise - qui est régi par ses propres codes génériques. Ce jeu s'inscrit dans une relation particulière avec un public d'amateurs déterminé, la cour des souverains de Norvège ou le grand public lettré islandais. Or, dès lors que les publics visés ne sont plus païens, et durant la période du "miracle islandais" tout le monde est chrétien, la représentation des croyances anciennes suppose une distance sinon critique du moins amusée, distance permettant justement à un projet littéraire de se développer plus ou moins librement à partir des sources les plus anciennes et les plus vénérables. Snorri Sturluson lui-même ne procède pas autrement dans son Edda; et dans les sagas, les exemples que nous pourrons prendre pour illustrer notre propos peuvent toujours être interprétés comme relevant d'une volonté de séduire, divertir et amuser le public avec des scènes reconstituées à la manière des temps anciens - nous en verrons des exemples.

\section{Visions}

La littérature norroise représente donc à sa manière un univers de croyances anciennes enracinées dans une appréhension païenne du monde. Il nous semble que les deux notions envisagées ici, visions et spectres, représentent en fait le même phénomène, le spectre étant un de ces êtres sortant de l'envers du décor pour se montrer aux vivants. Il se trouve que nous possédons par ailleurs en français un vocable d'origine normande qui pourrait nous intéresser, puisque "hanter" vient de l'ancien scandinave heimta. Le terme fait partie des mots que les Vikings ont apportés dans l'ancienne province de Neustrie devenue Normandie au $\mathrm{X}^{\mathrm{e}}$ siècle. Heimta signifie au départ "mener à la maison" ou "rentrer à la maison", et "hanter" en ancien français a le sens d'habiter ou fréquenter. En fait, le mot ne s'applique pas aux revenants dans les textes norrois, et le mot français semble avoir subi l'influence récente de l'anglais to haunt qui a la même origine, mais qui englobe le sens spécifique aux lieux fréquentés par des fantômes. Ce terme n'apporte donc pas de nuance très nouvelle par rapport aux mots d'origine latine dont nous nous contenterons ici.

Tout à la fois donc, les êtres sortant de l'invisible peuvent venir rencontrer les vivants, de même que plus rarement les vivants peuvent faire une incursion dans le monde du double. Dès lors, les uns et les autres sont vus, ou aperçus, selon des modalités de perception spéciale que l'on peut nommer "vision" pour employer un terme moderne. De quelle nature sont donc ces échanges, ces circulations entre monde des hommes vivants et monde du double? Il existe tout simplement des techniques permettant de franchir les limites. Le maître incontesté en est le dieu Odin (Ódinn), patron des magiciens, le créateur même de cet art magique qui permet de circuler librement de part et d'autre de la frontière, et d'en retirer un avantage matériel : connaissance du passé et du futur, contacts avec le peuple de l'autre 
monde pour intervenir sur la destinée des vivants, etc. Snorri Sturluson donne de l'origine de la magie une description saisissante au début de la Heimskringla ${ }^{4}$; ce que nous pouvons nommer magie recouvre en fait des pratiques diverses ${ }^{5}$ dont les sagas nous livrent de nombreux exemples en nous montrant des magiciens à l'œuvre. Le texte de Snorri, par contre, n'est pas très explicite à soi seul et demande à être éclairci. Quel rapport existe-t-il, en effet, entre ces pratiques magiques et le monde du double ? Il nous faut en vérité préciser comment l'individu lui-même est pensé, car nous touchons là une construction intellectuelle très éloignée de nos conceptions qui viennent de l'Antiquité gréco-latine et chrétienne.

L'individu est lui-même double ou multiple. Il existe tout d'abord une enveloppe physique extérieure, corporelle, périssable et même interchangeable - si l'on est capable par la magie odinique de se métamorphoser. En outre, un individu possède en lui une part d'un principe actif universel, nommé hugr, qu'on peut traduire par esprit vital. Il peut arriver qu'on ne le maîtrise plus si l'on tombe par exemple sous le coup d'un charme lancé par un magicien. Il existe même des hugir errants qui ne sont plus rattachés à un corps. Et il réside aussi en chacun une sorte de forme interne, le hamr (l'âme peut-on dire) qui est lié à la destinée de l'individu. Par des techniques magiques, cette âme peut sortir du corps et se libérer des contingences matérielles pour traverser l'espace et s'incarner dans d'autres enveloppes charnelles : dans la poésie eddique, les dieux ne cessent de se transformer, en animaux par exemple ; dans la légende de Sigurd (Sigurdr ou Siegfried), quand le héros rend visite à la valkyrie Brynhildr, il échange son apparence physique avec son ami Gunnar ${ }^{6}$. A l'intérieur du corps de Gunnar, le hamr est celui de Sigurd. Le phénomène des loups garous de nos folklores a la même origine. Par ailleurs, une personne donnée est aussi accompagnée tout au long de son existence par un double tutélaire nommé fylgja - le terme désignera l'ange gardien après la christianisation. Il est très dangereux de l'apercevoir, car il n'apparaît qu'à l'approche du trépas, quand l'individu est en train de basculer dans le monde du double. Il peut aussi se manifester sous forme animale. Enfin, il existe une divinité tutélaire qui est plutôt attachée à un groupe, la hamingja, car l'individu n'est jamais défini comme une singularité en dehors de tout lien social. Celle-ci représente positivement la chance qui protège une collectivité et peut apparaître sous la forme d'une entité féminine. Le terme désigne en islandais moderne la notion de bonheur. Et après la

4 Heimskringla (Cercle du monde) est le titre général donné ordinairement à l'histoire des rois de Norvège composée par Snorri Sturluson dans les années 1220-1230. Les origines mythiques de la Norvège sont présentées dans l'Ynglinga saga (Saga des Ynglingar), première pièce de cette fresque - voir les chapitres 6 et 7, trad. de François-Xavier DillmanN, Histoire des rois de Norvège, par Snorri Sturluson, Ière partie, Paris, Gallimard, 2000, p. 60-62. Nous n'indiquerons que des traductions françaises des textes mentionnés.

5 Voir pour une étude d'ensemble R. BOYER, Le monde du double : La magie chez les anciens Scandinaves.

6 Voir Völsunga saga (Saga des Völsungar), chap. 27 (trad. de R. BOYER, La saga de Sigurdr, ou la parole donnée, Paris, Éd. du Cerf, 1989, p. 246-248). 
mort ? malgré la disparition de l'enveloppe charnelle, la forme interne survit, et elle est même toujours visible, mais elle réside désormais dans un autre monde qui n'est pas régi par les lois ordinaires du temps et de l'espace.

Dans le monde du double, nos catégories ordinaires n'existent donc pas, et les êtres y ont accès au passé comme à l'avenir sans obstacle. La représentation mythologique a bien dessiné une géographie de l'au-delà avec, pour quelques guerriers élus, le séjour de la Valhöll (ou Walhalla) auprès d'Odin, et pour la plupart l'enfer de Hel. Mais cette construction résulte peut-être d'une rationalisation tardive due à des poètes et des mythographes, tel Snorri Sturluson ${ }^{7}$. Dans la vie courante telle que la montrent les sagas, l'autre monde est là présent à portée de la main.

Durant sa vie terrestre, l'individu suit un parcours tracé pour lui par des forces cachées qui le dépassent. La vie de chacun est conduite selon une dialectique subtile alliant volonté individuelle et consentement au destin omnipotent, lequel s'incarne aussi, dans le discours mythologique, dans des figures telles que les Nornes ou les Valkyries ${ }^{8}$. A l'arrière-plan de la société humaine, celles-ci surveillent la destinée de chacun. Les représentations que nous en avons dans les poèmes eddiques ou scaldiques ${ }^{9}$ apparaissent comme des mises en forme mythologiques, parfois même strictement littéraires, de croyances extrêmement répandues. Dans les sagas, sur un mode beaucoup plus prosaïque, une multitude de petits signes du destin viennent guider les personnages importants pour leur signifier qu'ils ne sont pas les seuls maîtres de leur destinée ${ }^{10}$. Normalement cachées dans le monde du double, ces entités divines apparaissent parfois dans les textes sous forme de visions.

Nous pensons par exemple au magnifique poème eddique du $\mathrm{XI}^{\mathrm{e}}$ siècle qui est inséré dans la Saga de Njáll le Brûlé, le Darradarljod (Lai de la lance $)^{11}$. Le texte dresse un tableau des divinités du destin à leur ouvrage en train de filer les destinées humaines; spectacle saisissant entrevu par un personnage sortant d'une bataille. Il est aussi possible, à l'inverse du chemin ordinaire, que certains de ces êtres de l'autre monde s'incarnent dans une enveloppe charnelle, telle la valkyrie Brynhildr dans la légende des Niflungar,

7 Voir dans l'Edda de Snorri la partie mythologique intitulée Gylfaginning (Mystification de Gylfi) (trad. de Fr.-X. Dillmann, Paris, Gallimard, 1991, p. 27-102).

8 Voir par exemple R. SIMEK, Dictionnaire de la mythologie germano-scandinave (trad. française de P. GuELPA), Paris, Éd. du Porte-Glaive, 1995, 2 vol. (original allemand : Lexikon der germanischen Mythologie, Stuttgart, A. Kröner Verlag, 1984).

9 Par exemple le Hrafnmál (Chant du corbeau) du scalde Thorbjörn Hornklofi (trad. de P. RENAULD-KRANTZ, Anthologie de la poésie nordique ancienne, Paris, Gallimard, 1964, p. 159-165).

10 Tel Gunnar de Hlídarendi, l'un des héros de la Brennu-Njáls saga (la Saga de Njáll le Brûlé, chap. 75-77) qui tombe de selle au montant où il doit quitter l'Islande où il est en danger; à la suite de ce signe, il ne part plus et mourra peu de temps après (Trad. de R. BOYER, Sagas islandaises, Paris, Gallimard, 1987, p. 1318-1324 ("Bibliothèque de la Pléiade").

11 Voir la Saga de Njáll le Brûlé, chap. 167 (trad. R. BOYER, Sagas islandaises, p. 14961498, ou L'Edda poétique, Paris, Fayard, 1992, p. 551-555). 
qui est punie par Odin pour avoir désobéi à ses ordres, et qui épouse le héros Gunnar tout en restant attachée à Sigurd ${ }^{12}$.

Tout nous ramène sans cesse à Odin, dont le portrait tracé par Snorri Sturluson ${ }^{13}$ fait apparaitre des pratiques importantes que nous retrouvons dans les sagas. Ainsi, le galdr ${ }^{14}$ (chant ou incantation) qui permet, par la parole, de communiquer avec l'autre monde, et d'agir de l'intérieur sur les êtres et les choses. Ce chant magique peut aussi s'intégrer dans un ensemble plus complexe de pratiques nommé sejdr, dont la Saga d'Eric le Rouge nous offre un bel exemple ${ }^{15}$. Ce passage au demeurant souvent commenté ${ }^{16}$, montre une magicienne à l'œuvre, une prophétesse qui vient au secours des Scandinaves de la colonie du Groënland. Maîtrisant parfaitement un art présenté comme féminin, elle fait accourir les esprits et communique avec eux. L'auteur de la saga étale tout son savoir magique dans une description détaillée à l'excès. Cet Islandais du XIII ${ }^{\mathrm{e}}$ siècle essaie de reconstituer une scène telle qu'elle a pu se dérouler vers l'an mil, et il aborde là un sujet délicat pour un chrétien du Moyen Âge. De ce fait, il eût sans doute été difficile pour lui d'en dire ou d'en montrer plus, et le poème magique interprété par la prophétesse n'est pas cité. La pratique du $s e j d r$ est d'ailleurs décrite comme ancienne et appelée à disparaître à un moment où le christianisme s'installe au-delà même de l'Islande. Malgré une part de construction littéraire, ce texte éclaire tout de même ce qu'a été la pratique de la voyance dans la Germanie païenne : quelques personnes sont capables de maitriser des techniques odiniques leur permettant de s'extraire de leur corps contingent et de passer dans l'autre monde pour entrer en contact avec des esprits, et accéder au passé et à l'avenir.

Un des plus anciens poèmes de l'Edda, qui ouvre majestueusement le fameux recueil du codex regius, s'appelle justement Völuspá (Prédiction de la voyante $)^{17}$; une prophétesse y retrace déjà toute l'histoire du monde en détail - c'est la source principale de l'Edda de Snorri. Ce texte présente donc une sorte de vision propre à l'autre monde, à la fois dominante et panoramique, l'expérience réalisée par la voyante consistant en quelque sorte à passer la tête derrière le monde apparent, comme nous l'avons vu avec le tableau des fileuses à la fin de la Saga de Njáll.

Chez les mythographes aussi, des individus ont le privilège de passer dans l'au-delà, par exemple en descendant momentanément en enfer. Saxo Grammaticus, clerc danois, a relaté dans les Gesta Danorum l'histoire de son pays des origines mythiques jusqu'au XII ${ }^{\mathrm{e}}$ siècle. Dans le premier livre ${ }^{18}$, le

\footnotetext{
12 Saga des Völsungar, chap. 20-31 (trad. R. BOYER, p. 230-261).

13 Voir la Saga des Ynglingar, chap. 2-9 (trad. Fr.-X. DillmanN, p. 56-64).

14 Galdrar au pluriel.

15 Eiriks saga rauda, chap. 4 (trad. R. BOYER, Sagas islandaises, p. 336-339).

16 Voir R. BOYER, Le monde du double, p. 16-19, et passim.

17 Trad. R. BOYER, L'Edda poétique, p. 532-549.

18 Gesta Danorum, I. $\S 14$ (trad. de Jean-Pierre Troadec, La Geste des Danois, Paris, Gallimard, 1995, p. 53-54)
} 
héros Hadingus est mené par une femme dans un lieu effrayant où des morts continuent à se battre. Il s'agit bien entendu d'une représentation de la Valhöll dont Hadingus a une vision avant l'heure, en sortant anormalement du monde des vivants auquel il appartient encore.

A titre d'hypothèse, sans doute risquée, nous serions tenté d'établir une proximité thématique entre ces visions furtives de l'au-delà et le genre littéraire médiéval de la vision qui a connu un grand succès dans le monde des clercs durant tout le haut Moyen Âge, avec des prolongements bien plus $\operatorname{tardifs}^{19}$. Les sources de ce genre littéraire sont parfaitement chrétiennes, puisque le voyage dans l'au-delà apparaît au sein de la littérature apocryphe qui complète le Nouveau Testament dans la culture médiévale. La descente du Christ aux enfers est racontée dans l'Évangile de Nicomède, et la Vision de saint Paul fixe le cadre de nombreuses descriptions de l'au-delà. Peu à peu, dans les visions postérieures, un cadre littéraire se stabilise, non sans rapport avec les visions païennes. En effet, souvent le visionnaire, au cours d'un sommeil ou d'une catalepsie, se dédouble ; son corps demeure inerte et son âme part à l'aventure dans l'au-delà. Elle va alors visiter des contrées inconnues qui présentent des apparences paradisiaques ou infernales. Parmi les plus célèbres de ces visions, plusieurs sont dues à des moines irlandais et semblent recycler des éléments issus de la tradition celtique. D'autres aussi ont été écrites en Germanie au sens large, peut-être sous l'influence des Irlandais, mais on peut y voir aussi malgré tout la résurgence de conceptions d'origine païenne, dans la Vision de Wettin, par exemple, moine de Reichenau (IX ${ }^{\mathrm{e}}$ siècle), dans celle de Godescalc $\left(\mathrm{XI}^{\mathrm{e}}\right.$ siècle, Schleswig), ou encore celle de Turchill (cistercien anglais du XIII ${ }^{\mathrm{e}}$ siècle). Les thèmes, l'imagerie et même le vocabulaire se retrouveront ensuite jusque dans la mystique rhénoflamande, chez Hildegarde de Bingen, et d'autres religieuses.

\section{Spectres}

Dans la littérature de sagas, l'autre monde se manifeste continuellement auprès des vivants ; c'est une expérience courante qui ne se produit jamais sans raison ni de façon anodine. Nous laisserons pour un temps de côté la question des revenants proprement dite, c'est-à-dire des morts qui retournent dans le monde des vivants où ils ne devraient pourtant plus paraitre. D'autres êtres au statut ambigu se manifestent en effet dans un contexte de danger pour prévenir les vivants - ces apparitions n'étant pas toujours malveillantes. Les personnages des sagas sont ainsi régulièrement avertis par des événements de toutes natures que le narrateur n'a même pas besoin de commenter, car ils sont explicites en eux-mêmes ; chacun est ensuite libre de tenir compte ou non de ces signes, mais de toute façon nul n'échappe à son destin.

19 Voir Alexandre MichA, Voyages dans l'au-delà, d'après des textes médiévaux (IV XIII ${ }^{e}$ siècles), Paris, Klincksieck, 1992. 
Prenons la Saga des Groënlandais. Plusieurs scènes se situent au Vínland, donc outre-atlantique, où une petite communauté scandinave a du mal à s'implanter dans des territoires mal connus, au bout du monde, presque à la frontière justement du monde visible et de l'au-delà. Les menaces concrètes viennent d'un peuple autochtone mystérieux nommé Skrcelingjar ${ }^{20}$. Lorsque ces derniers reviennent avec des produits à échanger, de façon tout à fait exceptionnelle, le double du personnage de Gudridr, sa fylgja, se montre sans rien dire. Cette présence indique un danger imminent, qui sera ainsi évité ${ }^{21}$.

Le cadre du songe se prête aussi tout particulièrement à la communication avec l'autre monde. L'esprit du dormeur est alors libre de franchir la frontière. Le motif n'est bien sûr pas propre à la Scandinavie ni à la Germanie. C'est même le cadre de beaucoup de visions mystiques au Moyen Âge. Comme nous l'avons vu, par un effet de dédoublement de la personne, le corps reste inerte alors que l'esprit veille et entre en communication avec des êtres venus de l'autre monde; au réveil, il en reste des traces dans la conscience du dormeur, sous forme de visions plus ou moins claires, qu'il est possible d'interpréter si l'on en a la capacité. Toutefois, dans les sagas nous ne trouvons pas de système interprétatif aussi élaboré que dans l'allégorie médiévale par exemple - même si un texte tel que la Queste del saint Graal est contemporain des sagas islandaises. Certains textes sont explicites, d'autres moins, preuve peut-être encore une fois de l'embarras du clerc chrétien face à des croyances d'origine païenne.

Parmi les chefs d'œuvre du genre, la Saga de Gísli Súrsson mérite une attention particulière, car le héros de ce récit est particulièrement touché par ce phénomène. Dans la dernière partie de sa vie, Gísli Súrsson, qui a commis un meurtre, vit banni dans une situation précaire, confronté à des dangers perpétuels $^{22}$. Il rêve alors de plus en plus souvent sous la forme d'obsessions harassantes qui l'empêchent même de dormir. Le contenu de ces visions oniriques est évoqué de façon peu claire dans des strophes scaldiques insérées au cour de la narration en prose, selon un procédé courant du genre de la saga. Ces rêves consistent en l'apparition de deux entités féminines, l'une rassurante, l'autre menaçante. On comprend bien que ces images mentales recoupent un enjeu littéraire : dramatiser le parcours tragique du héros qui vit dans l'incertitude au quotidien, ballotté entre l'espoir d'un répit (la première femme) et la certitude d'une mort prochaine (la seconde femme).

Ces strophes ont fait couler beaucoup d'encre ${ }^{23}$. Comment interpréter ces visions? Normalement un individu n'a qu'une seule fylgja, alors que Gísli voit deux femmes différentes. Faut-il adopter un point de vue chrétien et y voir la confrontation simpliste des forces du bien à celles du mal, puisque ce

20 Il peut s'agir d'Inuits ou d'Indiens. Voir le dossier de R. BOYER, Ísland, Græenland, Vinland, Essai sur le mouvement des Scandinaves vers l'ouest au Moyen Age, Paris, Éd. Arkhê, 2011.

21 Grolendinga saga, chap. 7 (trad. R. BOYER, Sagas islandaises, p. 368-371).

22 Gísla saga Súrssonar, chap. 30-34 (trad. R. BOYER, Sagas islandaises, p. 622-631).

23 Voir la synthèse de R. BOYER, ibid., p. 1676-1680. 
personnage a vécu au moment où l'Islande a adopté la foi chrétienne, ce qu'indiquent régulièrement les auteurs des sagas ? Sommes-nous dès lors sortis des croyances anciennes pour entrer dans une représentation purement littéraire de l'inconscient du héros ? La succession de ces visions semble illustrer de façon poignante l'incertitude qui définit l'existence du héros, faite d'oscillations entre moments de répit et moments de danger. Il est en tout cas plus facile d'interpréter des sagas explicitement chrétiennes; ainsi dans la Saga de saint Óláf de Snorri Sturluson ${ }^{24}$, le roi Óláfr Haraldsson, futur saint, est prévenu en rêve avant la bataille où il va trouver la mort, après laquelle les miracles se multiplieront ${ }^{25}$.

Il reste les revenants, registre extrêmement développé dans les sagas. La mort est par excellence le moment où s'opère une communication entre les deux mondes, parce que l'individu bascule de l'un dans l'autre ${ }^{26}$. Dans la tradition hérö̈que par exemple, telle qu'elle est illustrée dans la poésie des scaldes, quand sur le champ de bataille apparaissent les valkyries, il est clair que la dernière heure d'un héros est arrivée et qu'il ne va pas tarder à partir rejoindre Odin dans la Valhöl1 ${ }^{27}$. Toute vision trop nette de l'au-delà est aussi mauvais signe pour les personnages de saga ${ }^{28}$. Après le temps de la transition, du trépas, dans la majorité des cas, l'individu, réduit à sa forme interne mais conservant une apparence corporelle, disparaît définitivement du monde des vivants. Néanmoins, on rencontre aussi de nombreux exemples de morts qui reviennent soit d'eux-mêmes, soit parce qu'on les fait revenir sous l'effet de la magie. Parmi les revenants fréquents, on trouve ceux qui sont morts pour des causes anormales : assassinats injustes, suicides, mort due à la magie, noyades, etc. Reviennent aussi ceux qui ont abandonné derrière eux des affaires en plan qu'ils ne veulent pas laisser en l'état.

Le plus souvent, dans les sagas, il ne s'agit pas de revenants errants, ce qui relève d'une autre croyance, mais de morts retournant sur les lieux de leur vie quotidienne pour y poursuivre une action inachevée. Ainsi dans la Saga de Snorri le Godi, plusieurs chapitres mettent en scène des revenants innombrables dont les vivants ont bien du mal à se débarrasser ${ }^{29}$. Claude Lecouteux a dressé une véritable galerie de portraits, notamment à partir

24 Cette saga est la pièce centrale de l'histoire des rois de Norvège intitulée Heimskringla (voir supra note 4).

25 Óláfs saga helga, chap. 214 et 244-245 (trad. R. BOYER, La Saga de saint Óláf, Paris, Payot, 1983, p. 242 et 263-267).

26 Voir l'étude de R. BOYER, La mort chez les anciens Scandinaves, Paris, Les Belles Lettres, 1994

27 Tel le roi Hákon le Bon qui périt à la bataille de Svold en 961. Le scalde Eyvindr Skáldaspillir (Ruine-scaldes) raconte dans les Hákonarmál (Chant de Hákon) comment le roi accéda ainsi auprès d'Odin - Trad. de P. RENAULD-KrANTZ, Anthologie, p. 191-196.

28 Dans Eyrbyggja saga (Saga de Snorri le Godi, chap. 51), des signes inquiétants se manifestent pendant plusieurs jours dans la ferme de Fródá. Ainsi, pour commencer, une pluie de sang précède de peu la mort de Thorgunna (trad. R. BOYER, Sagas islandaises, p. 299-302).

29 Il s'agit toujours des événements de Fródá - Eyrbyggja saga, chap. 52-55 (ibid., p. 303308). 
d'extraits de sagas, dont celle que nous venons de $\operatorname{citer}^{30}$ : un mari jaloux ne veut pas que sa femme lui trouve un remplaçant, un propriétaire refuse de renoncer à ses biens et vient terroriser ceux qui les ont repris, un revenant rentre chez lui avec sa tête coupée sous le bras, certains reviennent sous forme aussi animale, si bien qu'on peut très normalement intenter un procès à un revenant, et ainsi de suite.

Le cadavre nécessite donc de strictes précautions, quels que soient les rites funéraires décrits : la tombe ou le tumulus, ou le bûcher, doivent être préparés selon les pratiques requises par la loi avec l'accompagnement des formules adéquates. Les auteurs de meurtre doivent ainsi, sous peine de poursuite, prendre en charge les frais d'inhumation, et certains cadavres sont parfois pourvus de chaussures spécialement attachées pour qu'ils puissent s'en aller $\operatorname{loin}^{31}$. On note aussi de nombreux cas où le mort, sans revenir parmi les hommes, continue de mener quelque temps une vie normale dans son tertre ${ }^{32}$, signe inquiétant.

Inversement, il existe aussi des circonstances dans lesquelles l'on a besoin de faire revenir un mort, en recourant aux techniques magiques dont nous avons déjà parlé. Une œuvre légendaire du $\mathrm{XIV}^{\mathrm{e}}$ siècle, la Saga de Hervör et $d u$ roi Heidrekr, recompose une matière héroïque remontant à un passé lointain, à partir de poèmes eddiques, sous la forme de grandes scènes spectaculaires. Dans l'une d'elles, Hervör, fille du roi Heidrekr, force son père à sortir de son tertre pour récupérer l'épée Tyrfingr, symbole de souveraineté $\mathrm{e}^{33}$. Ce genre de pratiques représente sans doute pour les Islandais chrétiens du Moyen Âge une caractéristique typique de la Scandinavie archaïque, qu'on peut alors reconstituer sous forme de fiction littéraire dans une visée de divertissement plus que d'édification historique.

Nous aurions pu bien évidemment citer en parallèle des textes anglais ou allemands, mais ils n'auraient pas montré une telle diversité en matière de visions et de spectres. Les clercs islandais, apparemment, ont pris plaisir à évoquer ces croyances sans les craindre outre mesure, sans les censurer fortement non plus ni les mettre en cause. Ils s'en amusent tout en étant fiers du passé de leurs ancêtres, se contentant d'en atténuer les aspects trop choquants. Leurs récits présentent l'intérêt de contenir des matériaux anciens de première main, même s'ils mêlent subtilement l'histoire et la fiction, dans un art strictement maîtrisé.

30 Fantômes et revenants au Moyen Age, p. 65 ss. et p. 189 ss.

31 Ce sont les "chaussures de Hel” mentionnées à l'occasion de l'enterrement de Vésteinn, beau-frère de Gísli Súrsson - Gísla saga Súrssonar, chap. 14 (trad. de R. BoyER, Sagas islandaises, p. 592).

32 Ainsi Gunnar de Hlídarendi (voir note 10), dans la Saga de Njáll le Brûlé, qui passe du bon temps dans son tertre après sa mort, en récitant des strophes poétiques - Brennu Njáls saga, chap. 78 (trad., R. BOYER, Sagas islandaises, p. 1324-1325).

33 Hervarar saga ok Heidreks konungs, chap. 4 (trad. R. BOYER, La Saga de Hervör et du roi Heidrekr, Paris, Berg International, 1988, p. 26-34). 
Nous citerons tout de même à titre d'exemple un passage du Dialogus miraculorum de Césaire de Heisterbach (XIII ${ }^{\mathrm{e}}$ siècle) ${ }^{34}$. Ce moine cistercien raconte une anecdote survenue dans l'archevêché de Mayence, qui peut être résumée comme suit : la maîtresse d'un prêtre meurt et on l'enterre, mais un chevalier la rencontre après son enterrement poursuivie par un chasseur infernal. Elle porte chemise et chaussures, et se désespère. Le chevalier essaie alors de la retenir pour la protéger, mais elle file et il lui arrache une partie de sa chevelure. Quand on ouvre la tombe pour vérifier le récit du chevalier, la morte n'a plus de cheveux ${ }^{35}$.

De tels témoignages sont là pour nous convaincre de la permanence des croyances tout au long du Moyen Âge, dans une ère donnée, bien après la christianisation, et malgré l'inlassable travail de l'Eglise en matière de conversion, qui il est vrai va changer de nature au XIII ${ }^{\mathrm{e}}$ siècle avec l'accentuation de la répression des superstitions et de la sorcellerie - ce qui nous entraîne vers une modernité sortant de notre propos.

34 Dialogus miraculorum, XII, 20.

35 Exemple repris également par Cl. LECOUTEUX, Fantômes et revenants au Moyen Age, p. $118-119$. 